\title{
Conectividade Funcional Cerebral Utilizando Técnicas de Imagens por Ressonância Magnética
}

\author{
Cerebral Functional Connectivity Using \\ Magnetic Resonance Imaging Techniques
}

\author{
Carlos E. G. Salmon ${ }^{1,2}$ (D), Renata F. Leoni ${ }^{1,2}$ (D) \\ ${ }^{1}$ InBrain, Departamento de Física, Faculdade de Filosofia, Ciências e Letras de Ribeirão Preto, \\ Universidade de São Paulo, Ribeirão Preto, Brasil \\ ${ }^{2}$ Núcleo de Apoio à Pesquisa em Física Médica, Universidade de São Paulo, Ribeirão Preto, Brasil
}

\begin{abstract}
Resumo
Nosso cérebro é reconhecido como uma rede complexa com propriedades topológicas de mundo pequeno: alta eficiência global, estrutura modular comunitária e elevado índice de agrupamento. Essa organização é preservada em diferentes escalas e para diferentes tipos de medidas de conectividade, especialmente ao se analisar a conectividade funcional em escala macroscópica. Neste artigo de revisão procuramos abordar aspectos metodológicos gerais para a estimativa da conectividade funcional (CF) cerebral humana a partir de imagens por ressonância magnética funcionais (IRMf). A CF é introduzida a partir de estudos prévios de neuroimagem funcional. Detalhes práticos e limitações da aquisição dos dados são discutidos considerando duas técnicas de aquisição: baseada no contraste dependente do nível de oxigenação do sangue e a marcação dos spins arteriais. Os principais passos para estimativa da CF a partir destas imagens são apresentados em duas etapas: pré-processamento e análise. Finalmente, diferentes aplicações dessas estimativas de CF cerebral na área da saúde são também apresentadas.
\end{abstract}

Palavras-chave: integração cerebral; imagem funcional por ressonância magnética; marcação dos spins arteriais.

\begin{abstract}
Our brain is recognized as a complex network with small world topological properties: high overall efficiency, modular community structure and high clustering index. Such organization is preserved at different scales and for different types of connectivity measures, especially when analyzing functional connectivity on a macroscopic scale. In this review, we seek to address general methodological aspects for the estimation of human brain functional connectivity (FC) from functional magnetic resonance imaging. FC is introduced from previous functional neuroimaging studies. Practical details and limitations of data acquisition are discussed considering two acquisition techniques: based on the Blood Oxygenation Level Dependent contrast and the Arterial Spin Labeling. The main steps to estimate FC from such images are presented in two steps: pre-processing and analysis. Finally, different applications of these brain FC estimates in the health area are also presented.
\end{abstract}

Keywords: brain integration; functional magnetic resonance imaging; arterial spin labeling.

\section{Introdução}

Entre as técnicas de neuroimagem, a ressonância magnética se destaca pela sua não invasividade e especialmente pela sua versatilidade para estudos de natureza estrutural ou funcional do cérebro humano. As aplicações em neurociências das imagens por ressonância magnética funcionais (IRMf) envolvem estudos de segregação e de integração funcional ${ }^{1,2}$. Em estudos de segregação são identificadas regiões do cérebro anatomicamente especializadas em alguns aspectos de uma determinada função cerebral.
Na abordagem de integração procura-se determinar como regiões cerebrais segregadas funcionalmente interagem durante a execução de determinada tarefa explicitada ou mesmo no repouso, isto é, como determinadas regiões se conectam. Ainda, duas grandes divisões metodológicas podem ser encontradas nos estudos de conectividade: avaliação de conectividade funcional (CF) e de conectividade efetiva ${ }^{2}$. A CF pode ser definida como a associação ou a dependência estatística entre séries temporais extraídas de regiões anatomicamente distantes, sem importar a causalidade ou a direção da conexão ${ }^{3}$. Já a conectividade efetiva 
procura explicar, mediante um modelo previamente desenhado, a influência que um sistema neural exerce sobre outro, seja no âmbito sináptico ou de população neuronal ${ }^{4}$. As análises de CF podem ser consideradas mais simples, pois são procurados padrões espaciais de conectividade e comparados estes entre grupos de indivíduos; porém nada é inferido sobre como acontece o acoplamento entre as diferentes regiões cerebrais.

O teste de hipótese na análise de CF normalmente envolve rejeitar a hipótese nula de independência estatística entre as séries temporais das diferentes regiões. Essas séries temporais podem ser obtidas sob diferentes paradigmas de aquisição e assumindo determinadas considerações. Diversos detalhes devem ser considerados para uma correta estimativa e interpretação da CF cerebral, não somente durante a aquisição da imagem, mas também no processamento dos dados.

Neste artigo procuramos abordar aspectos metodológicos gerais da aquisição das imagens e o processamento dos dados para a estimativa da conectividade funcional cerebral a partir de IRMf. Diferentes aplicações dessas estimativas de CF na área da saúde são também mencionadas.

Se consideramos os diferentes mecanismos de contraste das imagens por ressonância magnética, duas técnicas funcionais destacam-se: a técnica baseada no contraste dependente do nível de oxigenação do sangue (BOLD, blood oxygenation level dependent) e o método de marcação dos spins arteriais (ASL, arterial spin labeling). A seguir abordaremos separadamente ambas as técnicas de aquisição nos estudos de CF.

\section{BOLD}

As alterações observadas no sinal BOLD são decorrentes das variações locais na homogeneidade do campo magnético experimentado pelas moléculas de água nas vizinhanças dos capilares. Tais alterações são decorrentes das diferentes propriedades magnéticas das hemoglobinas nesses capilares. A oxihemoglobina (oxi-Hb) é diamagnética tendendo a perturbar menos a distribuição do campo magnético local; já a deoxihemoglobina (deoxi-Hb) é paramagnética, por isso perturba mais significativamente a homogeneidade local. Um aumento da atividade neuronal implica um consumo energético adicional que demanda oxigênio para o tecido cerebral. Essa demanda provoca um aumento não proporcional do fluxo sanguíneo e uma variação de concentração de oxigênio local, reduzindo a razão [deoxi-Hb]/[oxi-Hb] nos capilares. Essa redução local leva a um aumento da relaxação transversal afetada pela homogeneidade local (T2*) dos núcleos de água nas vizinhanças desses capilares, originando-se o contraste BOLD ${ }^{5}$. Dessa forma, é estabelecida certa relação entre a atividade neural e a resposta hemodinâmica detectada nas IRMf, embora tenham dinâmicas temporais bem diferentes. O sinal BOLD pode ser considerado uma amostragem filtrada e subamostrada do potencial de campo local que é uma medida direta das flutuações de atividade elétrica da população neuronal ${ }^{6}$. Ainda que de forma indireta, a análise desse sinal permite extrair informação sobre as interações de populações neuronais que supostamente têm lugar ao nível dos campos locais?

Embora existam variações na relaxação transversal intrínseca (T2) devido às alterações na concentração de oxigênio no sangue, o valor de T2* é mais afetado. Desse modo, sequências do tipo eco de gradiente (GE, gradient echo) são preferidas no lugar das sequências eco de spin para realçar o contraste BOLD, mesmo quando estas últimas são menos afetadas pelo efeito de grandes vasos ${ }^{8}$. Procurando aumentar a velocidade da aquisição de imagens com uma resolução espacial razoável, na ordem de poucos milímetros, o eco gerado pelo gradiente de campo é codificado com uma técnica conhecida como echo planar imaging (EPI). Assim, com a combinação GE-EPI são comumente adquiridas imagens de todo encéfalo com resolução isotrópica de 3 a 4 mm em aproximadamente 2 segundos em equipamentos comerciais amplamente usados na rotina clínica. Mais recentemente, com a introdução de equipamentos de 7 Tesla para uso em humanos e técnicas de reconstrução paralela, é possível adquirir imagens com resoluções submilimétricas em tempos menores ${ }^{9}$ ou com aumento de especificidade 8,10 .

Em estudos de IRMf usando o contraste BOLD e envolvendo a execução de tarefas cognitivas que demandam atenção, duas respostas são comumente observadas. Um conjunto de áreas corticais parietais e frontais mostra um incremento da atividade ${ }^{11}$; e outro conjunto, incluindo o cingulado posterior, o córtex pré-frontal medial e o córtex parietal medial e lateral, exibe uma redução da atividade ou "desativação"12. Essas diferenças ficam mais acentuadas na medida em que a demanda atencional da tarefa é incrementada. A partir de dados de tomografia de emissão de pósitrons, foi mostrada a existência de um conjunto de áreas no cérebro que no estado de repouso têm atividade contínua e espontânea, parcialmente suspensa durante a execução de tarefas externas ${ }^{13}$. Esse conjunto é atualmente definido como rede cerebral ${ }^{14}$. Esse tipo de evidência indica um modo padrão (default mode) de funcionamento cerebral e, assim, a total relevância dos estudos em estado de repouso para uma melhor compreensão de outras diferentes redes e uma definição clara dos termos ativação e desativação. Adicionalmente, os neurônios mostram alto nível de disparos espontâneos mesmo na ausência de tarefas específicas, transportando continuamente informações para outros neurônios. Nesse contexto, pode ser razoável especular sobre a ideia de que a conectividade funcional ajuda a manter os sistemas funcionais em um estado ativo, ajudando a melhorar o desempenho e seu tempo de reação ${ }^{15}$.

Os estudos de conectividade funcional em estado de repouso (resting state) examinam as correlações de flutuações espontâneas de baixa frequência $(<0.1 \mathrm{~Hz})$ no sinal BOLD. Biswal e colaboradores foram os primeiros a mostrar essas flutuações espontâneas coerentes no sistema somatomotor há quase 25 anos $^{16}$; isso foi estendido 
a outras redes de processamento: linguagem, visão, audição e cognitivas de alta ordem ${ }^{15}$. Aproveitando a disponibilidade atual de grandes bancos de imagens, diversas redes em estado de repouso têm sido reportadas com alto grau de reprodutibilidade ${ }^{17}$. Entre as mais relevantes, cinco delas identificadas pelos dois contrastes discutidos neste artigo são mostradas na Figura 1, a partir de um conjunto pequeno de participantes:

- modo padrão (contendo pré-cúneo, frontal medial, parie-

tal inferior, temporal inferior);

- visual primário;

- auditivo;

- saliência (contendo cingulado anterior, temporal bilateral, ínsula);

- motora

No geral, essas redes encontradas definem espacialmente áreas com funções e neuroanatomia semelhantes. Isto é, regiões moduladas de forma semelhante pelo estímulo, embora anatomicamente não vizinhas, exibem flutuações espontâneas correlacionadas decorrentes da atividade hemodinâmica ${ }^{15}$ e eletrofisiológica ${ }^{18}$, ainda na ausência do estímulo.

Em um experimento em estado de repouso geralmente o participante é introduzido no equipamento de ressonância e instruído a não pensar em nada, e em especial não adormecer. Não existe consenso se o participante deve manter os olhos fechados ou abertos. Neste último caso pode ser incluída uma tarefa de fixação visual, por exemplo olhar fixo em uma cruz, procurando reprodutibilidade e consistência nos dados.

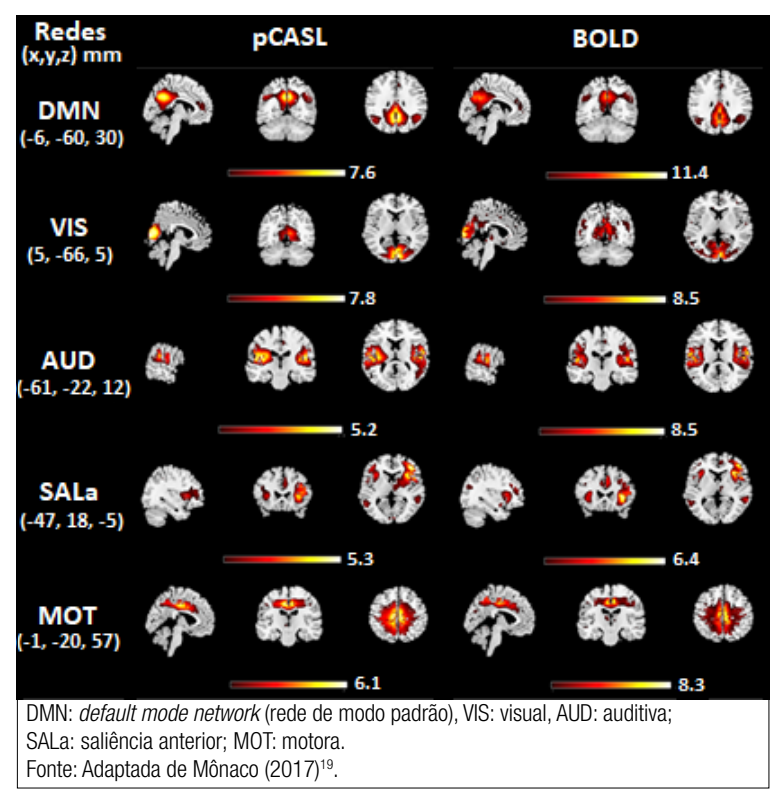

Figura 1. Redes cerebrais de repouso obtidas por análise de componentes independentes para imagens de perfusão adquirias por $A S L$ pseudocontínua (pCASL) e imagens BOLD. Barras de cores representam valores de $z$.
Embora os estudos pioneiros de conectividade funcional tenham sido baseados em flutuações do sinal BOLD que emergem espontaneamente durante o repouso ${ }^{16}$, existem também flutuações síncronas durante paradigmas de tarefas $^{19,20}$ e em estados variados de consciência ${ }^{21,22}$. O procedimento comum de medir flutuações síncronas em repouso não implica que os estados de repouso tenham um status especial que maximize a presença de flutuações coerentes em todos os sistemas. No entanto, como as flutuações espontâneas são frequentemente medidas em repouso, o método é constantemente referido como fcMRI do "estado de repouso" (R-fcMRI) e as redes cerebrais são identificadas como "redes de estado de repouso" (RSNs - resting state networks). A medição durante o repouso ou a fixação passiva tem a vantagem de minimizar as flutuações BOLD provocadas pela tarefa e é bastante simples de implementar ${ }^{23}$.

Entretanto, apesar de o contraste BOLD ser atualmente o método não invasivo mais usado para estudos de CF, ele é sensível a diferentes parâmetros hemodinâmicos (fluxo sanguíneo cerebral, FSC; volume sanguíneo cerebral e extração de oxigênio), o que aumenta a sensibilidade, mas reduz a especificidade. Adicionalmente, essa relação não é completamente conhecida em indivíduos afetados por doenças neurológicas. Por outro lado, o método ASL fornece mapas quantitativos específicos de FSC sem o uso de contrastes exógenos, possibilitando a avaliação da conectividade funcional cerebral ${ }^{24-26}$.

\section{Arterial Spin Labeling Funcional}

A ASL é uma técnica que utiliza a água presente no sangue arterial como agente de contraste endógeno difusível. Seu princípio baseia-se na diferenciação entre os spins fluindo com o sangue marcado magneticamente por um pulso de radiofrequência e os spins estacionários. À medida que os spins intravasculares marcados atingem os capilares e realizam trocas com o tecido por meio da barreira hematoencefálica, a magnetização do tecido é alterada e torna-se possível obter imagens cujo contraste é proporcional à perfusão sanguínea cerebral ${ }^{27,28}$. A partir dessas imagens ponderadas em perfusão, obtêm-se mapas quantitativos de FSC.

Há três métodos principais de marcação, contínuo (CASL - continuous ASL), pulsado (PASL - pulsed ASL) e pseudocontínuo (pCASL - pseudocontinuous ASL). CASL usa pulsos adiabáticos, constantes e longos (2 a 4 s), juntamente com um gradiente de campo magnético na direção do fluxo, para marcar um volume pequeno próximo à região de interesse ${ }^{29}$. Apesar da alta relação contraste-ruído e da alta eficiência de marcação, o sinal é reduzido devido à transferência de magnetização e há grande deposição de energia no sujeito ${ }^{27}$. Essas questões são minimizadas com a marcação de um volume relativamente grande de sangue, em uma região próxima às fatias de interesse, com um único pulso de RF curto (5 a 20 ms), que é o caso da PASL. Contudo, as relações contraste-ruído e sinal-ruído (RSR) são mais baixas ${ }^{30,31}$. Como alternativa, pCASL combina as 
vantagens dos métodos anteriores, CNR e eficiência de marcação altas com menos demanda de hardware e deposição de energia, sendo o método recomendado para o uso clínico ${ }^{31}$. Em pCASL, um trem de pulsos curtos de RF (aproximadamente $1 \mathrm{~ms}$ ) e gradientes de campo sincronizados são usados para inverter os spins do sangue fluindo por meio do plano de marcação. Mais detalhes sobre os métodos de marcação e quantificação do FSC podem ser encontrados na literatura recente $e^{31,32}$.

Além da avaliação quantitativa da perfusão sanguínea basal, as imagens de ASL obtidas ao longo do tempo podem revelar informações sobre a funcionalidade do cérebro tanto durante uma tarefa específica quanto em repouso ${ }^{33,34}$. Devido ao acoplamento entre perfusão regional, metabolismo da glicose e atividade neuronal ${ }^{35}$, flutuações no sinal de perfusão refletem alterações na atividade neuronal subjacente $^{36}$. É possível, então, identificar alterações de FSC devido a um estímulo ou uma tarefa, reconhecer oscilações espontâneas de baixa frequência nos mapas de FSC adquiridos em repouso, assim como determinar parâmetros de rede, realizando análises de conectividade funcional similares àquelas realizadas com o contraste BOLD $24,37-39$. Trabalhos recentes mostraram que a ASL fornece poder estatístico, reprodutibilidade e caracterização das flutuações de baixa frequência comparáveis ao contraste BOLD ${ }^{25,40}$. Além disso, foi mostrada uma similaridade regional entre os mapas de conectividade funcional obtidos por ASL e BOLD em repouso em relação à conectividade positiva; já o padrão espacial da conectividade negativa foi mais variável, sendo de menor extensão quando obtido por imagens de $A S L{ }^{41}$. Entretanto, a origem e a interpretação dessas correlações negativas ainda estão em discussão.

A maioria dos estudos de conectividade funcional com IRM durante uma tarefa ou durante o repouso tem usado o contraste BOLD. Apesar de seu uso bastante difundido, ele tem algumas desvantagens: não especificidade à atividade neuronal, devido à contribuição de ruído fisiológico ao mecanismo de contraste; não especificidade espacial, devido à contribuição de sinal proveniente de veias de drenagem; não fornecimento de uma medida direta e quantitativa da função

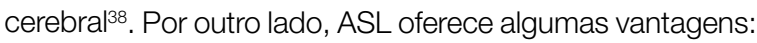
maior especificidade espacial à atividade neuronal, pois o sinal tem origem no nível dos capilares; quantificação do FSC, que é um parâmetro crítico na avaliação da viabilidade do tecido cerebral; medidas dinâmicas e simultâneas de FSC e sinal BOLD, permitindo a avaliação da taxa metabólica de oxigênio; menor variabilidade entre sujeitos da amplitude de ativação; menos suscetível a artefatos em regiões de altos gradientes de susceptibilidade magnética ${ }^{30,42}$.

Entretanto, as imagens de ASL apresentam baixa RSR, que contribui para séries de imagens de perfusão com baixas RSR e estabilidade temporais. Para reduzir esse problema, há um consenso de que o melhor método de aquisição a ser usado é o de marcação pseudocontínua com leitura tridimensional e supressão de sinal de fundo ${ }^{31}$. As aquisições tridimensionais aumentam a RSR espacial, enquanto a supressão de sinal de fundo melhora RSR e estabilidade temporais das séries de imagens, mesmo em aquisições bidimensionais ${ }^{43-45}$.

Além disso, como as imagens são adquiridas com métodos rápidos de leitura o sinal sofre atenuação devido aos efeitos de relaxação $T 2^{*}$, que são similares às flutuações fisiológicas observadas em imagens BOLD ${ }^{46}$. Para melhor quantificação de $\mathrm{CBF}$, esses efeitos podem ser minimizados com a utilização de tempos ao eco (TEs) curtos, geralmente entre 8 e 15 ms; porém, a intensidade do sinal é reduzida ${ }^{38}$. Uma alternativa é adquirir as imagens de ASL com leitura de eco duplo (dual-echo ASL, DE-ASL) para otimizar a quantificação do CBF e a análise funcional. Imagens adquiridas com TE curto apresentam mais ponderação em perfusão, enquanto as adquiridas com TE longo apresentam mais efeitos típicos do contraste BOLD ${ }^{46}$. Estudos recentes têm mostrado a viabilidade do método na avaliação da conectividade funcional em repouso e com tarefas motora e visual, e sua relação com o FSC basal ${ }^{47,48}$. Entretanto, esses estudos não avaliaram o efeito de TE no padrão espacial das redes de repouso e da conectividade funcional. A Figura 2 mostra a diferença espacial da rede motora obtida por séries temporais de CBF usando 2 valores de TE (9 ms e $28 \mathrm{~ms}$ ) durante uma tarefa motora realizada por apenas uma mão.

Por fim, a resolução temporal da técnica é também intrinsecamente baixa. Tempos de repetição (TR) de 2 a 4 segundos são os mais utilizados; dessa forma, um par de imagens controle/marcada é adquirido de 4 a 8 segundos. Apesar dos novos métodos para acelerar a aquisição dos dados, a ASL esbarra em uma questão fisiológica. É necessário que o sangue marcado chegue à região de interesse para perfundir o tecido; isso depende da velocidade do FSC de cada sujeito, limitando o TR mínimo.

\section{Pré-Processamento das Imagens}

Independentemente da técnica e do paradigma de aquisição usados, o processo de aquisição de dados tem duração relativamente longa, da ordem de minutos. Dessa forma, é inevitável a existência de uma variabilidade indesejável nas séries temporais adquiridas, seja por motivos intrínsecos da

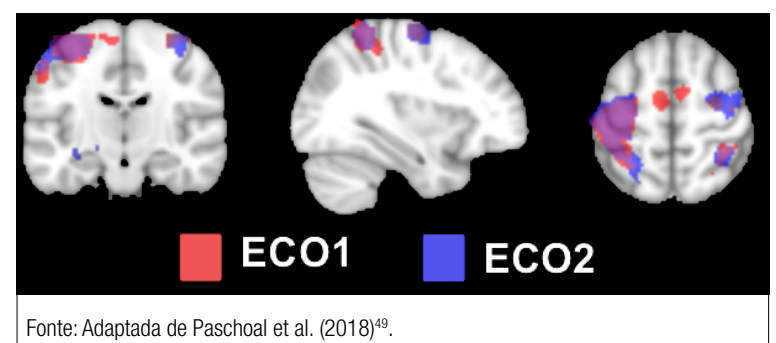

Figura 2. Rede cerebral motora obtida por uma série temporal de mapas de fluxo sanguíneo cerebral em um grupo de sujeitos jovens saudáveis, comparando os resultados para imagens obtidas com dois valores de tempo ao eco $\left(\mathrm{TE}_{1}=9 \mathrm{~ms}\right.$ e $\mathrm{TE}_{2}=28 \mathrm{~ms}$ ). 0 coeficiente de similaridade foi de 0.67 . 
técnica de aquisição ou por ruídos fisiológicos. Visando remover esses "artefatos" no sinal de interesse, diversos passos de pré-processamento são geralmente aplicados ${ }^{23,50,51}$ :

- Correção de tempo-fatia: visa corrigir a diferença temporal entre as fatias de um determinado volume, pois elas não são adquiridas ao mesmo tempo dentro de uma repetição (em um TR);

- Correção de movimento: procura reduzir o efeito dos movimentos involuntários da cabeça do participante durante a aquisição, mediante transformações de corpo rígido aplicadas ao volume da imagem. Após este passo é esperado que todos os volumes da aquisição dinâmica estejam corregistrados entre si;

- Normalização, segmentação e corregistro: diferentemente dos outros, este passo é executado sob as imagens anatômicas concomitantemente adquiridas e com maior resolução espacial que as funcionais. Neste passo essas imagens são levadas ao espaço de um determinado atlas para a definição de regiões anatômicas, classificadas segundo o tipo de tecido cerebral e corregistradas com as imagens funcionais para usar nestas últimas as informações anteriores obtidas (regiões anatômicas e composição tecidual);

- Remoção linear de variáveis de confusão: procura eliminar diversos efeitos transientes indesejáveis com a aplicação de um modelo linear geral. Como covariáveis no nível do sujeito são normalmente incluídos o sinal médio da substância branca, o sinal médio do fluido cérebro espinhal, os parâmetros da transformação aplicada na correção de movimento e parâmetros separadamente adquiridos que caracterizem ruídos fisiológicos devido à respiração ou ao ciclo cardíaco. Neste passo alguns autores incorporam também como variável de confusão o sinal de todo o volume em estado basal para eliminar uma componente de ruído térmico associado à fisiologia do participante. Se por um lado a remoção desse sinal melhora a RSR temporal das séries de mapas de FSC e o resultado dos testes de confiabilidade ${ }^{52,53}$, ela também aumenta o número de correlações negativas entre regiões cerebrais ainda não completamente entendidas ${ }^{54}$. Além disso, estudos sugerem que as flutuações de baixa frequência desse sinal global podem estar associadas com o sincronismo de todos os neurônios ${ }^{55}$. Portanto, ainda não há um consenso em relação à inclusão ou não deste último fator;

- Remoção de tendências: elimina elementos de não estacionariedade na série temporal usando correções polinomiais de diversas ordens;

- Filtragem temporal: objetiva definir o intervalo de frequência de interesse, entre 0.01 e $0.1 \mathrm{~Hz}$ no caso do sinal BOLD, mediante a aplicação de um filtro passa-banda comum;

- Suavização espacial: traz um aumento significativo da RSR com a aplicação de um filtro espacial, porém com redução da resolução espacial;

- Normalização: passo requerido em estudo de grupo ou análise de segundo nível, para levar todos os volumes a um espaço geométrico comum.
Alguns passos de pré-processamento aqui mencionados são optativos, sua ordem de aplicação depende do pesquisador e a maior parte está implementada em diversas ferramentas: CONN no SPM ${ }^{56}$, FEAT no $\mathrm{FSL}^{57} \mathrm{e}$ FATCAT no AFN|58.

O método de correção de artefatos de movimento em imagens BOLD se baseia na minimização das diferenças de intensidade em uma série de imagens. Entretanto, na técnica ASL, imagens controles e marcadas são adquiridas de maneira intercalada, e apresentam intensidades diferentes devido à marcação dos spins arteriais. Essa diferença pode ser interpretada erroneamente como movimento pelo algoritmo de realinhamento. Assim, recomenda-se realizar o realinhamento das imagens controles e marcadas separadamente, e depois um corregistro adicional de todas as imagens utilizando um método baseado em informação mútua ${ }^{59}$.

Além disso, em ASL, presume-se que a quantidade de sinal relacionada à perfusão sanguínea é proporcional à diferença entre as imagens controles e marcadas. Assim, os mapas de FSC são calculados usando as imagens de perfusão e abordagens matemáticas ${ }^{60}$. No entanto, diferentes métodos de subtração têm sido usados (Figura 3). O primeiro, a subtração simples, calcula a diferença de sinal entre um par de imagens controle/marcada para obter uma imagem ponderada em perfusão. Embora simples e amplamente utilizado, ele não filtra ruído fisiológico ${ }^{61}$. Portanto, outros métodos foram propostos ${ }^{54}$ : surround subtraction, que utiliza uma imagem média controle (ou marcada) calculada a partir de duas imagens obtidas antes e depois da imagem marcada (ou controle), atenuando ruídos de alta frequência, como o cardíaco; subtração sinc, que consiste em uma correção da diferença temporal entre aquisições de imagens controle e marcada, agindo como um filtro passa-banda; running subtraction, que aumenta o número de pontos nas séries temporais do FSC e melhora a RSR temporal.

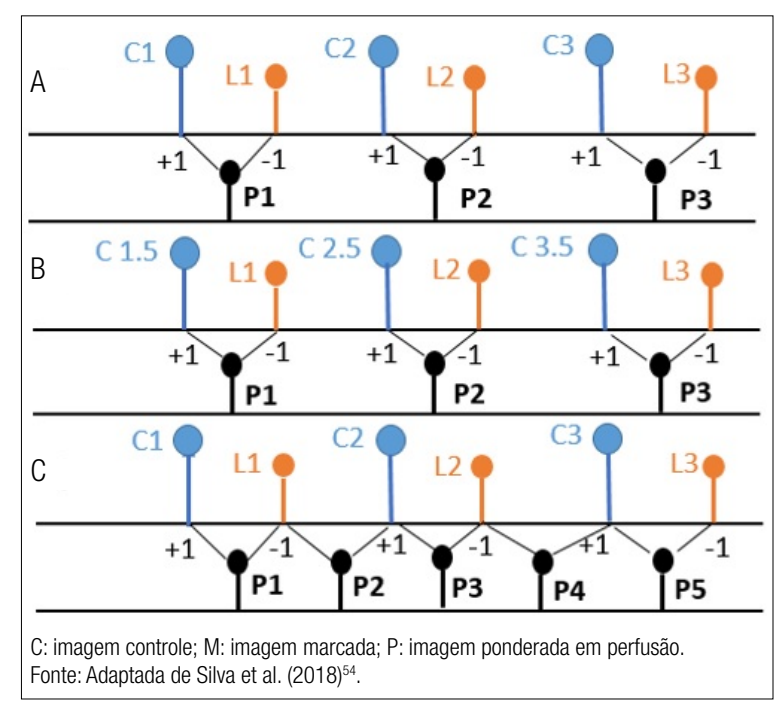

Figura 3. Representação esquemática dos métodos de subtração (A) simples, (B) sinc e (C) running para obter as imagens ponderadas em perfusão. 


\section{Métodos de Análise}

Diferentes métodos de análise têm sido empregados para avaliar a CF independentemente do mecanismo de contraste explorado (BOLD ou ASL). Essa avaliação pode ser na forma de uma métrica representando uma dependência estatística entre séries temporais, trials ou blocos; ou na forma de mapas espaciais após agrupar voxels ou regiões com base também na série temporal latente.

Na procura por dependência estatística entre séries temporais, diferentes métricas têm sido usadas: correlação simples $^{16}$ ou parcial|62, coerência cruzada ${ }^{63}$, informação mútua inicialmente aplicada em estudos de EEG ${ }^{64}$ e correlação canônica ${ }^{65}$. Nesses métodos são comparadas as séries temporais por pares, sendo uma delas tomada como modelo e proveniente de uma região semente, por este motivo, no contexto de CF, são chamados métodos dependentes de modelo ou de semente. A métrica de associação mais usada, pela sua facilidade de implementação e interpretação, é a correlação simples com o coeficiente de correlação de Pearson definido entre duas séries $\left(x_{A}, x_{B}\right)$ (Equação 1):

$\rho=\frac{\operatorname{Cov}\left(x_{A}, x_{B}\right)}{\sqrt{\operatorname{Cov}\left(x_{A}, x_{A}\right) \cdot \operatorname{Cov}\left(x_{B}, x_{B}\right)}}$

Aqui, Cov indica a covariância entre as séries. Esse coeficiente representa a associação simultânea entre as séries temporais. Associações não simultâneas podem existir e são consideradas em outras métricas, por exemplo, modelos autorregressivos, porém classificadas na maior parte da literatura como estimativas de conectividade efetiva e assim fora do contexto do presente artigo.

Existem diferentes estratégias para a definição da região semente, que pode ser um único voxel ou toda uma região de interesse (ROI - region of interest). Essa $\mathrm{ROI}$ pode ser definida previamente a partir de um experimento envolvendo um paradigma orientado a uma tarefa específica, fato que pode dificultar o procedimento de aquisição, apesar de contribuir para a especificidade. O resultado da aplicação do método de semente resulta em uma matriz de conectividade ou de associação cuja interpretação depende da estratégia de escolha das sementes e das ROls associadas a um determinado atlas anatômico.

Como alternativa aos métodos de semente, existem os métodos livres de modelo, nos quais são explorados padrões de conexão funcional no cérebro todo fazendo uso da independência estatística espacial ou temporal dos dados, sem a necessidade de especificar, a priori, um modelo temporal explícito. Entre esses métodos temos: análise de componente independente (ICA - independent component analysis) ${ }^{66}$, análise de componente principal (PCA - principal component analysis) ${ }^{67}$ e algoritmos de clusterização, como a homogeneidade regional|68 ou a clusterização hierárquica ${ }^{69}$. Esses métodos livres de modelo podem ser de difícil interpretação, porém os resultados usando dados de imagens funcionais adquiridos durante o repouso têm sido similares aos obtidos com métodos de semente ${ }^{23}$.
Nas avaliações de CF a partir de aquisições em estado de repouso é assumida a estacionariedade da série, isto é, seus principais parâmetros estatísticos permanecem constantes no tempo. Condição localmente necessária em paradigmas do tipo bloco considerando a duração destes. Já em aquisições relacionadas a evento, a estacionariedade não é requerida. A inferência de alterações de conectividade relacionadas à tarefa pode ser obtida dividindo amostras de dados de diferentes períodos de tarefas em diferentes condições (depois de levar em consideração o atraso hemodinâmico) e depois calculando as métricas de conectividade de cada condição separadamente.

Atualmente, a maioria dos estudos envolve um grande número de indivíduos, neste caso é feita uma análise de grupo ou análise de segunda ordem, em que sobre os resultados de CF podem ser aplicados modelos estatísticos procurando representar a matriz de conectividade com uma significância estatística. Nesse caso, outras variáveis podem ser usadas, por exemplo, valores t ou z. Para uma visualização mais intuitiva a matriz de conectividade obtida é geralmente representada na forma de um mapa sobreposto a uma imagem anatômica. Na Figura 4 diversos mapas são apresentados usando diferentes regiões sementes para obter as redes de repouso a partir de dados de BOLD e ASL em poucos indivíduos. Tal resultado pode ser contrastado com o apresentado na Figura 1 usando um método livre de modelo e estatística z.

A teoria de grafos permite abstrair propriedades de sistemas complexos, como o cérebro, para ser quantitativamente caracterizados e mapeados, possibilitando tomar medidas simples que podem ser usadas para estudar as tendências globais que definem a arquitetura normal do cérebro e sua variabilidade entre sujeitos ${ }^{23}$. Um paralelo entre as propriedades topológicas das redes complexas de mundo pequeno e das redes cerebrais foi sugerido no começo do século por Sporns et al. ${ }^{70}$; diversos trabalhos têm confirmado essa proposta ${ }^{15,50}$. O conjunto de CF do

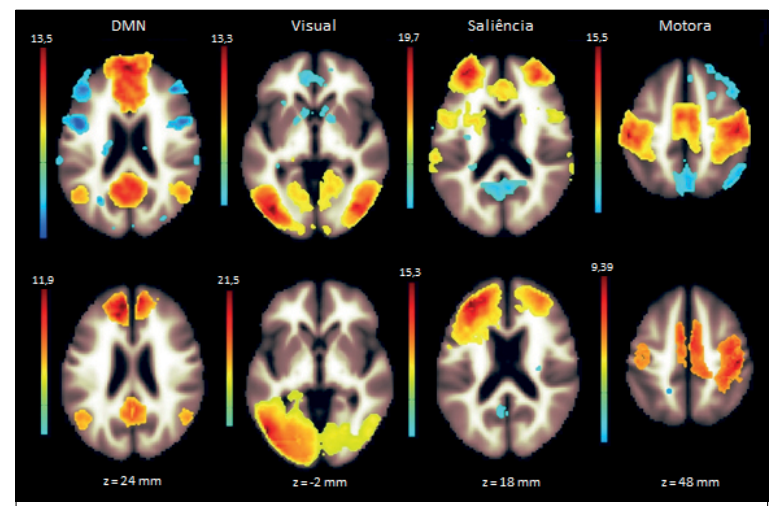

Fonte: Adaptada de Mônaco (2017) ${ }^{19}$.

Figura 4. Redes cerebrais de repouso obtidas por análise baseada em sementes para as imagens BOLD (linha superior) e de ASL (linha inferior). As barras de cores representam os valores de $t$. 
cérebro pode ser considerado um grafo $G=(V, E)$, no qual $\checkmark$ representa a coleção de nodos refletindo as regiões cerebrais ou os neurônios e E, as conexões (ou arestas) funcionais entre essas regiões, sinapses ou projeções axonais. O nível de conectividade funcional entre duas regiões define a ponderação da rede e pode ser computado pelas métricas anteriormente comentadas. A rede pode ser binarizada, isto é, não ponderada, a partir da definição de certo limiar dependente do problema. Entre as propriedades topológicas mais utilizadas, encontramos: eficiência, coeficiente de agrupamento ou de clusterização, centralidade, modularidade e vulnerabilidade.

\section{Aplicações}

Diversos estudos têm sido reportados avaliando a influência de diferentes variáveis na conectividade cerebral baseada no contraste BOLD em indivíduos saudáveis: sexo $^{71}$, idade ${ }^{51,72}$, inteligência ${ }^{73}$, ingestão de psicoativos ${ }^{74}$, estados meditati$\operatorname{vos}^{75}$ e espessura cortical ${ }^{51}$. No caso do envelhecimento, existe maior número de conexões com aumento de CF com a idade quando comparadas com as reduções de CF (Figura 5); tal fato tem sido atribuído à presença de mecanismos compensatórios.

A CF tem sido utilizada como indicador global da integridade do sistema cerebral submetido a uma determinada

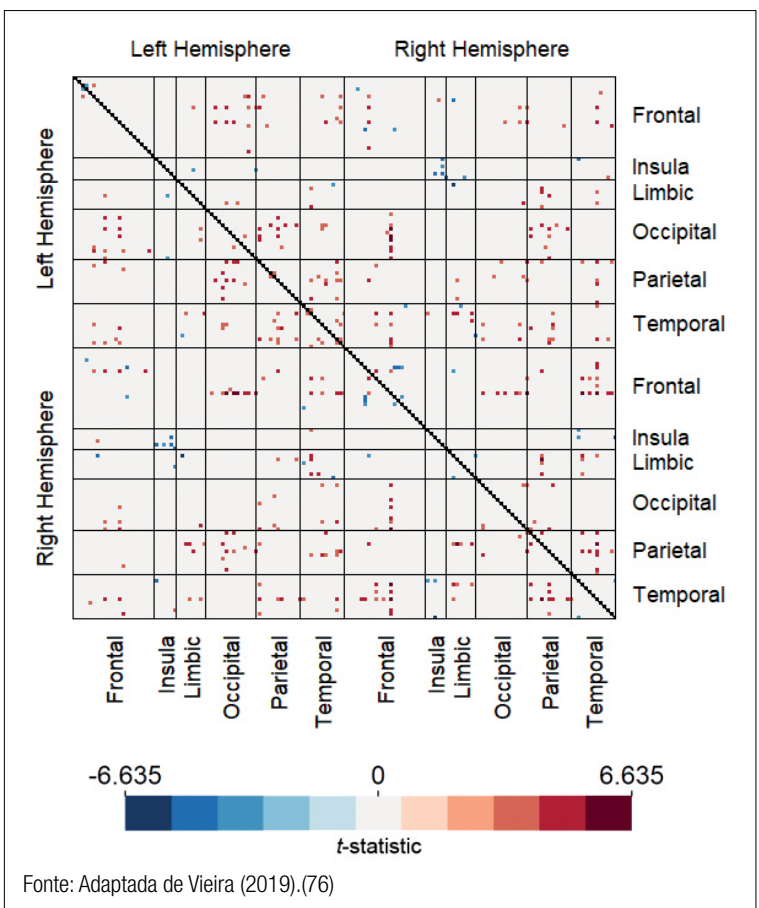

Figura 5. Efeito da idade na conectividade funcional bivariada estimada usando o coeficiente de correlação de Pearson em dados BOLD de uma amostra de 327 individuos. As cores representam os valores de t, em que vermelho indica associação positiva e azul, negativa. doença, principalmente avaliando os parâmetros topológicos da rede complexa definida pelas regiões anatômicas parceladas. Em pacientes com doença de Alzheimer foi observada, por alguns pesquisadores, uma diminuição do coeficiente de clusterização e da eficiência, sugerindo uma degradação dos atributos de rede de mundo pequeno, porém os resultados não são consensuais ${ }^{77}$. Adicionalmente, Greicius et al. mostraram que a CF na rede padrão em pacientes com Alzheimer é reduzida ${ }^{78}$. Em pacientes com esquizofrenia foi reportada uma perda de simetria, parâmetros topológicos próximos aos de uma rede randômica e certa perda de organização hierárquica ${ }^{77}$. Reduções de CF ou de extensão de redes de repouso foram relatadas em numerosas desordens neuropsiquiátricas como autismo, ADHD e depressão ${ }^{22}$. A definição das redes de repouso também tem sido usada na localização de regiões eloquentes sem a necessidade de um experimento que envolva execução de tarefas. Essa localização é de grande utilidade para planejamento cirúrgico no caso de pacientes que não colaboram voluntária ou involuntariamente.

Aproveitando as vantagens da ASL, estudos recentes têm mostrado alterações de conectividade funcional e suas relações com FSC basal e desempenho cognitivo em diferentes casos, como no desenvolvimento cerebral em crianças e adolescentes, no envelhecimento saudável e em pacientes com doença de Parkinson, esquizofrenia e síndrome da fatiga crônica ${ }^{39,79-84}$.

Diferentemente do contraste BOLD, ao utilizar dados de ASL para avaliar o efeito do envelhecimento na conectividade, predominam as áreas com redução de CF com o aumento da idade (Figura 6), como reportadas em um estudo recente de CF com $\mathrm{ASL}^{84}$. Essas regiões incluem o polo frontal, o córtex pré-frontal, o córtex orbitofrontal, o lobo temporal direito, o giro central, o giro supramarginal e o giro angular esquerdo.

A Figura 7 mostra a distribuição espacial de duas redes cerebrais de repouso, rede de modo padrão (DMN) e executiva central (CEN), de pacientes com esquizofrenia usando dados de ASL. Comparando com um grupo de sujeitos saudáveis com idade pareada, observa-se redução da extensão de ambas as redes no grupo de pacientes. Essa diferença foi relacionada com a redução da capacidade

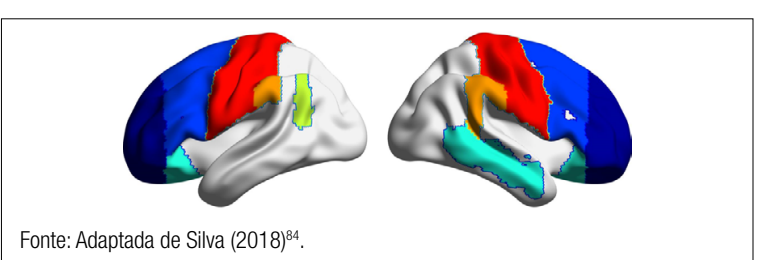

Figura 6. Regiões cerebrais que apresentaram redução significativa da conectividade funcional a partir de dados de arterial spin labeling (ASL) em função da idade: polo frontal, córtex pré-frontal e córtex orbitofrontal (azul), lobo temporal direito (verde), giro central (vermelho), giro supramarginal (laranja) e giro angular esquerdo (amarelo). 


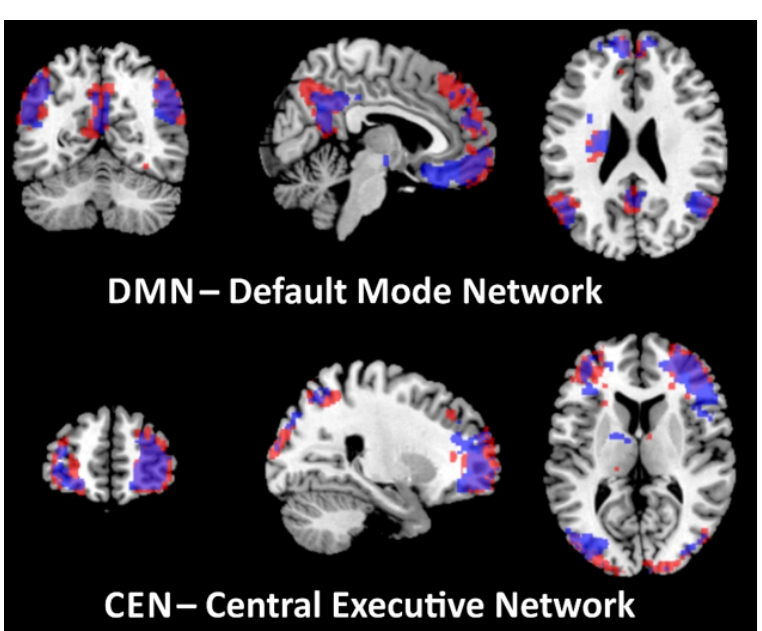

Figura 7. Redes de modo padrão (DMN, default mode network) e executiva central (CEN, central executive network) detectadas com dados de arterial spin labeling (ASL) de pacientes com esquizofrenia, em azul, e indivíduos saudáveis, em vermelho, mostrando a diferença na distribuição espacial.

desses pacientes em se envolverem com autoprocessamento durante atividades não direcionadas e aos déficits de memória de trabalho.

\section{Conclusão}

Diversas métricas de conectividade cerebral têm sido descritas, levando a modelar o cérebro como uma rede complexa em diferentes escalas espaciais. A conectividade funcional se destaca pela sua simplicidade, mesmo tendo uma interpretação limitada. A CF representa a dependência estatística entre séries temporais extraídas de regiões anatomicamente distantes, sem considerar a causalidade ou direção da conexão. No caso das IRMf e em escala macroscópica, ela pode ser extraída a partir de dados de sinal BOLD ou ASL em estado de repouso, porém alguns cuidados são requeridos na aquisição e no processamento dos dados.

O sinal BOLD tem alta sensibilidade a variações hemodinâmicas. A especificidade do sinal de ASL intrinsecamente relacionado ao metabolismo cerebral e à atividade neuronal torna a técnica muito interessante para obtenção de séries temporais de FSC, permitindo a avaliação de um único parâmetro hemodinâmico. No entanto, devido à relação sinal-ruído e a resoluções espaciais e temporais baixas das imagens obtidas, o estudo da CF com flutuações de FSC ainda está em desenvolvimento em relação à aquisição e ao processamento das imagens.

Embora a associação da CF com diversas variáveis de interesse biológico tenha sido mostrada, a correta interpretação dessas associações ainda é um desafio. Esse desafio deve-se fundamentalmente a nosso limitado conhecimento sobre o funcionamento cerebral integrado nas diferentes escalas espaciais e o significado indireto das variáveis medidas in vivo.

\section{Agradecimentos}

Agradecemos à Fundação de Amparo à Pesquisa do Estado de São Paulo (FAPESP), ao Conselho Nacional de Desenvolvimento Científico e Tecnológico (CNPq) e à Coordenação de Aperfeiçoamento de Pessoal de Nível Superior (CAPES), o apoio financeiro.

\section{Referências}

1. Zeki S, Shipp S. The functional logic of cortical connections. Nature. 1988;335(6188):311-7. https://doi.org/10.1038/335311a0

2. Friston KJ. Functional and effective connectivity: a review. Brain Connect. 2011;1(1):13-36. https://doi.org/10.1089/brain.2011.0008

3. Friston $\mathrm{KJ}$, Frith $\mathrm{CD}$, Fletcher $\mathrm{P}$, Liddle PF, Frackowiak RS. Functional topography: multidimensional scaling and functional connectivity in the brain. Cereb Cortex. 1996;6(2):156-64. https://doi.org/10.1093/ cercor/6.2.156

4. Breakspear M. "Dynamic" connectivity in neural systems: theoretical and empirical considerations. Neuroinformatics. 2004;2(2):205-26. https://doi. org/10.1385/Nl:2:2:205

5. Ogawa S, Lee TM, Kay AR, Tank DW. Brain magnetic resonance imaging with contrast dependent on blood oxygenation. Proc Natl Acad Sci U S A. 1990;87(24):9868-72. https://dx.doi.org/10.1073\%2Fpnas.87.24.9868

6. Logothetis NK, Pauls J, Augath M, Trinath T, Oeltermann A. Neurophysiological investigation of the basis of the fMRI signal. Nature. 2001;412(6843):150-7. https://doi.org/10.1038/35084005

7. Fox MD, Snyder AZ, Vincent JL, Corbetta M, Van Essen DC, Raichle ME. The human brain is intrinsically organized into dynamic, anticorrelated functional networks. Proc Natl Acad Sci U S A. 2005;102(27):9673-8. https://doi.org/10.1073/pnas.0504136102

8. Yacoub E, Van De Moortele PF, Shmuel A, Uğurbil K. Signal and noise characteristics of Hahn SE and GE BOLD fMRl at $7 \mathrm{~T}$ in humans. Neuroimage. 2005;24(3):738-50. https://doi.org/10.1016/j.neuroimage.2004.09.002

9. Heidemann RM, Ivanov D, Trampel R, Fasano F, Meyer H, Pfeuffer J, et al. Isotropic submillimeter fMRI in the human brain at $7 \mathrm{~T}$ : combining reduced field-of-view imaging and partially parallel acquisitions. Magn Reson Med. 2012;68(5):1506-16. https://doi.org/10.1002/mrm.24156

10. Boyacioğlu R, Schulz J, Koopmans PJ, Barth M, Norris DG. Improved sensitivity and specificity for resting state and task fMRI with multiband multi-echo EPI compared to multi-echo EPI at $7 \mathrm{~T}$. Neuroimage. 2015;119:352-61. https://doi.org/10.1016/j.neuroimage.2015.06.089

11. Cabeza R, Nyberg L. Imaging cognition II: An empirical review of 275 PET and fMRI studies. J Cogn Neurosci. 2000;12(1):1-47.

12. Gusnard DA, Raichle ME. Searching for a baseline: functional imaging and the resting human brain. Nat Rev Neurosci. 2001;2(10):685-94. https:// doi.org/10.1038/35094500

13. Raichle ME, MacLeod AM, Snyder AZ, Powers WJ, Gusnard DA, Shulman GL. A default mode of brain function. Proc Natl Acad Sci U S A. 2001;98(2):676-82. https://doi.org/10.1073/pnas.98.2.676

14. Greicius MD, Krasnow B, Reiss AL, Menon V. Functional connectivity in the resting brain: a network analysis of the default mode hypothesis. Proc Natl Acad Sci U S A. 2003;100(1):253-8. https://doi.org/10.1073/ pnas. 0135058100

15. van den Heuvel MP, Hulshoff Pol HE. Exploring the brain network: a review on resting-state fMRI functional connectivity. Eur Neuropsychopharmacol. 2010;20(8):519-34. https://doi.org/10.1016/j.euroneuro.2010.03.008

16. Biswal $B$, Yetkin FZ, Haughton VM, Hyde JS. Functional connectivity in the motor cortex of resting human brain using echo-planar MRI. Magn Reson Med. 1995;34(4):537-41. 
17. Miller KL, Alfaro-Almagro F, Bangerter NK, Thomas DL, Yacoub E, Xu J, et al. Multimodal population brain imaging in the UK Biobank prospective epidemiological study. Nat Neurosci. 2016;19(11):1523-36. https://doi. org/10.1038/nn.4393

18. Brookes MJ, Woolrich M, Luckhoo H, Price D, Hale JR, Stephenson $\mathrm{MC}$, et al. Investigating the electrophysiological basis of resting state networks using magnetoencephalography. Proc Natl Acad Sci U S A. 2011;108(40):16783-8. https://doi.org/10.1073/pnas.1112685108

19. Mônaco LM. Conectividade funcional cerebral no estado de repouso através de técnicas complementares de Imagens por Ressonância Magnética [tese]. Ribeirão Preto: Universidade de São Paulo; 2017.

20. Newton AT, Morgan VL, Gore JC. Task demand modulation of steadystate functional connectivity to primary motor cortex. Hum Brain Mapp. 2007;28(7):663-72. https://doi.org/10.1002/hbm.20294

21. Greicius MD, Kiviniemi V, Tervonen O, Vainionpää V, Alahuhta S, Reiss $\mathrm{AL}$, et al. Persistent default-mode network connectivity during light sedation. Hum Brain Mapp. 2008;29(7):839-47. https://doi.org/10.1002/ hbm.20537

22. Horovitz SG, Braun AR, Carr WS, Picchioni D, Balkin TJ, Fukunaga M, et al. Decoupling of the brain's default mode network during deep sleep. Proc Natl Acad Sci U S A. 2009;106(27):11376-81. https://doi.org/10.1073/ pnas.0901435106

23. Van Dijk KR, Hedden T, Venkataraman A, Evans KC, Lazar SW, Buckner RL. Intrinsic functional connectivity as a tool for human connectomics: theory, properties, and optimization. J Neurophysiol. 2010;103(1):297-321. https://doi.org/10.1152/jn.00783.2009

24. Liang X, Connelly A, Calamante F. Graph analysis of resting-state ASL perfusion MRI data: nonlinear correlations among CBF and network metrics. Neuroimage. 2014;87:265-75. https://doi.org/10.1016/j. neuroimage.2013.11.013

25. Jann K, Gee DG, Kilroy E, Schwab S, Smith RX, Cannon TD, et al. Functional connectivity in BOLD and CBF data: similarity and reliability of resting brain networks. Neuroimage. 2015;106:111-22. https://doi.org/10.1016/j. neuroimage.2014.11.028

26. Mayhew SD, Mullinger KJ, Bagshaw AP, Bowtell R, Francis ST. Investigating intrinsic connectivity networks using simultaneous BOLD and CBF measurements. Neuroimage. 2014;99:111-21. https://doi.org/10.1016/j. neuroimage.2014.05.042

27. Petersen ET, Zimine I, Ho YC, Golay X. Non-invasive measurement of perfusion: a critical review of arterial spin labelling techniques. $\mathrm{Br} \mathrm{J}$ Radiol. 2006;79(944):688-701. https://doi.org/10.1259/bjr/67705974

28. Jahng GH, Li KL, Ostergaard L, Calamante F. Perfusion Magnetic Resonance Imaging: A Comprehensive Update on Principles and Techniques. Korean J Radiol. 2014;15(5):554-77. https://doi.org/10.3348/kjr.2014.15.5.554

29. Dai W, Garcia D, de Bazelaire C, Alsop DC. Continuous flow-driven inversion for arterial spin labeling using pulsed radio frequency and gradient fields. Magn Reson Med. 2008;60(6):1488-97. https://doi.org/10.1002/mrm.21790

30. Wong EC, Buxton RB, Frank LR. Implementation of quantitative perfusion imaging techniques for functional brain mapping using pulsed arterial spin labeling. NMR Biomed. 1997;10(4-5):237-49.

31. Alsop DC, Detre JA, Golay X, Günther M, Hendrikse J, Hernandez-Garcia $L$, et al. Recommended implementation of arterial spin-labeled perfusion MRI for clinical applications: A consensus of the ISMRM perfusion study group and the European consortium for ASL in dementia. Magn Reson Med. 2014;73(1):102-16. https://doi.org/10.1002/mrm.25197

32. Hernandez-Garcia L, Lahiri A, Schollenberger J. Recent progress in ASL. Neuroimage. 2019;187:3-16. https://doi.org/10.1016/j. neuroimage.2017.12.095

33. Chuang KH, van Gelderen P, Merkle H, Bodurka J, Ikonomidou VN, Koretsky AP, et al. Mapping resting-state functional connectivity using perfusion MRI. Neuroimage. 2008;40(4):1595-605. https://doi.org/10.1016/j. neuroimage.2008.01.006
34. Jann K, Orosz A, Dierks T, Wang DJ, Wiest R, Federspiel A. Quantification of network perfusion in ASL cerebral blood flow data with seed based and ICA approaches. Brain Topogr. 2013;26(4):569-80. https://doi.org/10.1007/ s10548-013-0280-3

35. Hoge RD, Pike GB. Oxidative metabolism and the detection of neuronal activation via imaging. J Chem Neuroanat. 2001;22(1-2):43-52.

36. Dai W, Varma G, Scheidegger R, Alsop DC. Quantifying fluctuations of resting state networks using arterial spin labeling perfusion MRI. J Cereb Blood Flow Metab. 2016;36(3):463-73. https://doi.org/10.1177/0271678X15615339

37. Liang $X, Z$ Zu $Q, H e Y$, Yang Y. Coupling of functional connectivity and regional cerebral blood flow reveals a physiological basis for network hubs of the human brain. Proc Natl Acad Sci U S A. 2013;110(5):1929-34. https://doi. org/10.1073/pnas.1214900110

38. Chen JJ, Jann K, Wang DJ. Characterizing Resting-State Brain Function Using Arterial Spin Labeling. Brain Connect. 2015;5(9):527-42. https://doi. org/10.1089/brain.2015.0344

39. Oliveira IAF, Guimarães TM, Souza RM, dos Santos AC, Machado-deSousa JP, Hallak JEC, et al. Brain functional and perfusional alterations in schizophrenia: an arterial spin labeling study. Psychiatry Res Neuroimaging. 2018;272:71-8. https://doi.org/10.1016/j.pscychresns.2017.12.001

40. Federspiel A, Müller TJ, Horn H, Kiefer C, Strik WK. Comparison of spatial and temporal pattern for fMRI obtained with BOLD and arterial spin labeling. J Neural Transm (Vienna). 2006;113(10):1403-15. https://doi. org/10.1007/s00702-006-0434-5

41. Viviani R, Messina I, Walter M. Resting state functional connectivity in perfusion imaging: correlation maps with BOLD connectivity and resting state perfusion. PLoS One. 2011;6(11):e27050. https://doi.org/10.1371/ journal.pone.0027050

42. Aguirre GK, Detre JA, Zarahn E, Alsop DC. Experimental design and the relative sensitivity of BOLD and perfusion fMRI. Neuroimage. 2002;15(3):488-500.

43. Wang Y, Moeller S, Li X, Vu AT, Krasileva K, Ugurbil K, et al. Simultaneous multi-slice Turbo-FLASH imaging with CAIPIRINHA for whole brain distortionfree pseudo-continuous arterial spin labeling at 3 and 7 T. Neuroimage. 2015;113:279-88. https://doi.org/10.1016/j.neuroimage.2015.03.060

44. Vidorreta M, Wang Z, Rodríguez I, Pastor MA, Detre JA, FernándezSeara MA. Comparison of $2 \mathrm{D}$ and $3 \mathrm{D}$ single-shot ASL perfusion $\mathrm{fMR}$ sequences. Neuroimage. 2013;66:662-71. https://doi.org/10.1016/j. neuroimage.2012.10.087

45. Ghariq E, Chappell MA, Schmid S, Teeuwisse WM, van Osch MJP. Effects of background suppression on the sensitivity of dual-echo arterial spin labeling MRI for BOLD and CBF signal changes. Neuroimage. 2014;103:316-22. https://doi.org/10.1016/j.neuroimage.2014.09.051

46. Lu H, Donahue MJ, van Zijl PC. Detrimental effects of BOLD signal in arterial spin labeling $\mathrm{fMRl}$ at high field strength. Magn Reson Med. 2006;56(3):546-52. https://doi.org/10.1002/mrm.20976

47. Storti SF, Boscolo Galazzo I, Montemezzi S, Menegaz G, Pizzini FB. Dualecho ASL contributes to decrypting the link between functional connectivity and cerebral blow flow. Hum Brain Mapp. 2017;38(12):5831-44. https:// doi.org/10.1002/hbm.23804

48. Storti SF, Galazzo IB, Pizzini FB, Menegaz G. Dual-echo ASL based assessment of motor networks: a feasibility study. J Neural Eng. 2018;15(2):026018. https://doi.org/10.1088/1741-2552/aa8b27

49. Paschoal AM, Paiva FF, Leoni RF, editors. Simultaneous assessment of CBF and brain function through dual-echo Arterial Spin Labeling. in: 5th BRAINN Congress; 2018; Campinas, Brazil. Campinas; 2018.

50. Pamplona G. Conectividade funcional no cérebro: Uma análise das associações com desempenho intelectual e atenção sustentada usando imagens por ressonância magnética [tese]. São Paulo: Universidade de São Paulo; 2014.

51. Vieira BH. Estudo da conectividade funcional cerebral em regiões com redução da espessura cortical associadas ao envelhecimento sadio [dissertação]. Ribeirão Preto: Universidade de São Paulo; 2018. 
52. Wang Z. Improving cerebral blood flow quantification for arterial spin labeled perfusion MRI by removing residual motion artifacts and global signal fluctuations. Magn Reson Imaging. 2012;30(10):1409-15. https:// doi.org/10.1016/j.mri.2012.05.004

53. Jann K, Smith RX, Rios Piedra EA, Dapretto M, Wang DJ. Noise Reduction in Arterial Spin Labeling Based Functional Connectivity Using Nuisance Variables. Front Neurosci. 2016;10:371. https://doi.org/10.3389/ fnins.2016.00371

54. Silva JPS, Mônaco LDM, Paschoal AM, Oliveira Í, Leoni RF. Effects of global signal regression and subtraction methods on resting-state functional connectivity using arterial spin labeling data. Magn Reson Imaging. 2018;51:151-7. https://doi.org/10.1016/j.mri.2018.05.006

55. Bright MG, Murphy K. Is fMRI "noise" really noise? Resting state nuisance regressors remove variance with network structure. Neuroimage. 2015;114:158-69. https://doi.org/10.1016/j.neuroimage.2015.03.070

56. Whitfield-Gabrieli S, Nieto-Castanon A. Conn: a functional connectivity toolbox for correlated and anticorrelated brain networks. Brain Connect. 2012;2(3):125-41. https://doi.org/10.1089/brain.2012.0073

57. Woolrich MW, Ripley BD, Brady M, Smith SM. Temporal autocorrelation in univariate linear modeling of FMRI data. Neuroimage. 2001;14(6):1370-86. https://doi.org/10.1006/nimg.2001.0931

58. Taylor PA, Saad ZS. FATCAT: (an efficient) Functional and Tractographic Connectivity Analysis Toolbox. Brain Connect. 2013;3(5):523-35. https:// doi.org/10.1089/brain.2013.0154

59. Wang Z, Aguirre GK, Rao H, Wang J, Fernández-Seara MA, Childress AR, et al. Empirical optimization of ASL data analysis using an ASL data processing toolbox: ASLtbx. Magn Reson Imaging. 2008;26(2):261-9. https://doi.org/10.1016/j.mri.2007.07.003

60. Buxton RB. Quantifying CBF with arterial spin labeling. J Magn Reson Imaging. 2005;22(6):723-6. https://doi.org/10.1002/jmri.20462

61. Warnock G, Özbay PS, Kuhn FP, Nanz D, Buck A, Boss A, et al. Reduction of BOLD interference in pseudo-continuous arterial spin labeling: towards quantitative fMRI. J Cereb Blood Flow Metab. 2018;38(5):847-56. https:// doi.org/10.1177/0271678X17704785

62. Hampson M, Peterson BS, Skudlarski P, Gatenby JC, Gore JC. Detection of functional connectivity using temporal correlations in MR images. Hum Brain Mapp. 2002;15(4):247-62.

63. Sun FT, Miller LM, D'Esposito M. Measuring interregional functional connectivity using coherence and partial coherence analyses of fMRI data. Neuroimage. 2004;21(2):647-58. https://doi.org/10.1016/j. neuroimage.2003.09.056

64. Jeong J, Gore JC, Peterson BS. Mutual information analysis of the EEG in patients with Alzheimer's disease. Clin Neurophysiol. 2001;112(5):827-35.

65. Worsley K, Charil A, Lerch J, Evans A, editors. Connectivity of anatomical and functional MRI data. In: IEEE International Joint Conference on Neural Networks; 2005. 2005.

66. Kiviniemi V, Kantola JH, Jauhiainen J, Hyvärinen A, Tervonen 0. Independent component analysis of nondeterministic $\mathrm{fMRI}$ signal sources. Neuroimage. 2003;19(2 Pt 1):253-60.

67. Friston KJ, Frith CD, Liddle PF, Frackowiak RS. Functional connectivity: the principal-component analysis of large (PET) data sets. J Cereb Blood Flow Metab. 1993;13(1):5-14.

68. Zang Y, Jiang T, Lu Y, He Y, Tian L. Regional homogeneity approach to fMRI data analysis. Neuroimage. 2004;22(1):394-400. https://doi. org/10.1016/j.neuroimage.2003.12.030

69. van den Heuvel M, Mandl R, Pol H. Normalized group cut group clustering of resting-state fMRI data. PLoS One. 2008;3(4):e2001. https://doi. org/10.1371/journal.pone.0002001
70. Sporns 0, Tononi G, Edelman GM. Theoretical neuroanatomy: relating anatomical and functional connectivity in graphs and cortical connection matrices. Cereb Cortex. 2000;10(2):127-41. https://doi.org/10.1093/ cercor/10.2.127

71. Tian $L$, Jiang $T$, Wang $Y$, Zang $Y$, He $Y$, Liang $M$, et al. Altered restingstate functional connectivity patterns of anterior cingulate cortex in adolescents with attention deficit hyperactivity disorder. Neurosci Lett. 2006;400(1-2):39-43. https://doi.org/10.1016/j.neulet.2006.02.022

72. Ferreira LK, Busatto GF. Resting-state functional connectivity in normal brain aging. Neurosci Biobehav Rev. 2013;37(3):384-400. https://doi. org/10.1016/j.neubiorev.2013.01.017

73. Pamplona GS, Santos Neto GS, Rosset SR, Rogers BP, Salmon CE. Analyzing the association between functional connectivity of the brain and intellectual performance. Front Hum Neurosci. 2015;9:61. https://doi.org/10.3389/ fnhum.2015.00061

74. Fontes F. Alterações da default mode network provocadas pela ingestão de Ayahuasca investigadas por ressonância magnética funcional [dissertação]. Natal: Universidade Federal do Rio Grande do Norte; 2012.

75. Brewer JA, Worhunsky PD, Gray JR, Tang YY, Weber J, Kober H. Meditation experience is associated with differences in default mode network activity and connectivity. Proc Natl Acad Sci U S A. 2011;108(50):20254-9. https:// doi.org/10.1073/pnas.1112029108

76. Vieira BH, Garrido Salmon CE. A principled multivariate intersubject analysis of generalized partial directed coherence with Dirichlet regression: Application to healthy aging in areas exhibiting cortical thinning. J Neurosci Methods. 2019;311:243-52. https://doi.org/10.1016/j. jneumeth.2018.10.033

77. Bullmore E, Sporns 0. Complex brain networks: graph theoretical analysis of structural and functional systems. Nat Rev Neurosci. 2009;10(3):186-98. https://doi.org/10.1038/nrn2575

78. Greicius MD, Srivastava G, Reiss AL, Menon V. Default-mode network activity distinguishes Alzheimer's disease from healthy aging: evidence from functional MRI. Proc Natl Acad Sci U S A. 2004;101(13):4637-42. https://doi.org/10.1073/pnas.0308627101

79. Fernández-Seara MA, Mengual E, Vidorreta M, Castellanos G, Irigoyen $J$, Erro $E$, et al. Resting state functional connectivity of the subthalamic nucleus in Parkinson's disease assessed using arterial spin-labeled perfusion fMRI. Hum Brain Mapp. 2015;36(5):1937-50. https://doi. org/10.1002/hbm.22747

80. Boissoneault J, Letzen J, Lai S, O'Shea A, Craggs J, Robinson ME, et al. Abnormal resting state functional connectivity in patients with chronic fatigue syndrome: an arterial spin-labeling fMRI study. Magn Reson Imaging. 2016;34(4):603-8. https://doi.org/10.1016/j.mri.2015.12.008

81. Boissoneault J, Letzen J, Lai S, Robinson ME, Staud R. Static and dynamic functional connectivity in patients with chronic fatigue syndrome: use of arterial spin labelling fMRI. Clin Physiol Funct Imaging. 2018;38(1):128-37. https://doi.org/10.1111/cpf.12393

82. Cui LB, Chen G, Xu ZL, Liu L, Wang HN, Guo L, et al. Cerebral blood flow and its connectivity features of auditory verbal hallucinations in schizophrenia: A perfusion study. Psychiatry Res Neuroimaging. 2017;260:53-61. https:// doi.org/10.1016/j.pscychresns.2016.12.006

83. Liu F, Duan Y, Peterson BS, Asllani I, Zelaya F, Lythgoe D, et al. Resting state cerebral blood flow with arterial spin labeling MRI in developing human brains. Eur J Paediatr Neurol. 2018;22(4):642-51. https://doi. org/10.1016/j.ejpn.2018.03.003

84. Silva JPS. Avaliação dos efeitos do envelhecimento na hemodinâmica cerebral por imagens de ressonância magnética [tese]. Ribeirão Preto: Universidade de São Paulo; 2018. 